\title{
Protocol for developing the reporting guidelines for radiological case reports: Case Report for Radiology statement
}

\author{
Mengshu Wang ${ }^{1,2 \#}$, Ying Zhu ${ }^{3,4,5 \#}$, Xufei Luo ${ }^{6 \#}$, Xiaojuan Xiao ${ }^{7}$, Ling Wang ${ }^{6}$, Meng Lv $^{8,9,10}$, Qi Wang ${ }^{5,11}$, \\ Yaolong Chen ${ }^{1,12,13,14}$, Junqiang Lei ${ }^{2,15,16}$, Jinhui Tian ${ }^{1,14}$ \\ ${ }^{1}$ Evidence-Based Medicine Center, School of Basic Medical Sciences, Lanzhou University, Lanzhou, China; ${ }^{2}$ Department of Radiology, The First \\ Hospital of Lanzhou University, Lanzhou, China; ${ }^{3}$ The Michael G. DeGroote Cochrane Canada Center, McMaster University, Hamilton, ON, \\ Canada; ${ }^{4}$ The MacGRADE Center, McMaster University, Hamilton, ON, Canada; ${ }^{5}$ Health Research Methods, Evidence, and Impact (HEI), \\ McMaster University, Hamilton, ON, Canada; ${ }^{6}$ School of Public Health, Lanzhou University, Lanzhou, China; ${ }^{7}$ Department of Radiology, The \\ Eighth Affiliated Hospital, Sun Yat-sen University, Shenzhen, China; ${ }^{8}$ Department of Nephrology, Children's Hospital of Chongqing Medical \\ University, Chongqing, China; ${ }^{9}$ National Clinical Research Center for Child Health and Disorders, Ministry of Education Key Laboratory of \\ Child Development and Disorders, China International Science and Technology Cooperation Base of Child Development and Critical Disorders, \\ Chongqing, China; ${ }^{10}$ Chongqing Key Laboratory of Pediatrics, Chongqing, China; ${ }^{11}$ McMaster Health Forum, McMaster University, Hamilton, \\ ON, Canada; ${ }^{12}$ Institute of Health Data Science, Lanzhou University, Lanzhou, China; ${ }^{13}$ World Health Organization Collaboration Center for \\ Guideline Implementation and Knowledge Translation, Lanzhou, China; ${ }^{14}$ Key Laboratory of Evidence Based Medicine and Knowledge Translation \\ of Gansu Province, Lanzhou University, Lanzhou, China; ${ }^{15}$ Intelligent Imaging Medical Engineering Research Center of Gansu Province, Lanzhou, \\ China; ${ }^{16}$ Accurate Image Collaborative Innovation International Science and Technology Cooperation Base of Gansu Province, Lanzhou, China \\ \#These authors contributed equally to this work. \\ Correspondence to: Professor Jinhui Tian. Evidence-Based Medicine Center, School of Basic Medical Sciences, Lanzhou University, No. 199, \\ Donggang West Road, Chengguan District, Lanzhou 730000, China. Email: tianjh@lzu.edu.cn.
}

Background: In radiology, case reports play an important role in the presentation of a new disease or an unusual form of a common disease using radiological images. Radiology practitioners can refer to the CAse REport (CARE) statement to write and improve the quality of case reports; however, some CARE items are not applicable to the field of radiology. This protocol seeks to describe the methods and processes used to develop CARE extensions for radiology.

Methods: We plan to extend the existing CARE guidelines to radiological case reports. We will follow the steps recommended by the Enhancing the QUAlity and Transparency Of health Research (EQUATOR) network to develop the CAse Report for Radiology (CARR) statement and checklist for the reporting of case reports. The working group will constitute a multidisciplinary international team of experts, including methodologists, content experts (radiologists and clinicians), journal editors, and possibly consumer representatives. We will discuss and generate a list of initial items based on the CARE statement. Two to three rounds of the Delphi survey will be administered and an online consensus meeting will be held to reach a consensus and develop the final CARR checklist. The full reporting guidelines should be finalized within 1.5 years.

Discussion: The annual number of published radiological case reports has increased over the past 20 years; however, the quality of reporting still needs to be improved. Our protocol envisages the process and methodology for the development of the CARR guidelines, which we anticipate will be available soon and will help radiology practitioners.

Trial Registration: We have registered the protocol on the EQUATOR network (https://www.equatornetwork.org/library/reporting-guidelines-under-development/reporting-guidelines-under-development-forobservational-studies/\#CARR)

Keywords: Reporting guideline; CARE; case report; radiology 
Submitted Dec 14, 2021. Accepted for publication Jan 13, 2022.

doi: 10.21037/atm-21-7028

View this article at: https://dx.doi.org/10.21037/atm-21-7028

\section{Introduction}

A clinical case report describes observations from an individual medical case in detail, such as the diagnosis, treatment and medication $(1,2)$. Case reports have been proven to be helpful in recognizing new diseases, generating hypotheses for future research, and guiding healthcare and treatment toward individualization and customization $(2,3)$. In radiology, case reports play an important role in presenting a new disease or an unusual form of a common disease using radiological images (4). The annual number of published radiological case reports has increased over the past 20 years (5). However, the quality of the published case reports in radiology is inconsistent. A systematic assessment of the quality of case reports revealed that technical and editorial errors were frequent. Common problems in case reports include a lack of description of patient symptoms, a failure to identify the main outcomes, and a failure to draw conclusions $(1,2,4)$. Thus, reporting in case reports needs to be improved in several ways.

Lennon suggested that case reports should adhere to a systematic approach and be presented in a uniform manner, which will help future evaluations of case reports on similar topics (3). To address this issue, Gagnier et al. developed the CAse REport (CARE) Statement and Checklist, reporting guidelines for case reports (2). The CARE guidelines consist of a 36-item checklist that provides a comprehensive framework to enhance the transparency and accuracy of reporting $(2,6)$. CARE items are a group of checklists that are required to follow for those writing case reports. The CARE guidelines have been endorsed and recommended by many journals $(7,8)$. In addition, researchers around the world have extended the CARE guidelines into specific fields of clinical research. For example, Lavergne et al. adapted the CARE guideline and developed a 114-item checklist tailored to case reports of extracorporeal treatment in poisonings (9). Nagendrababu et al. developed the Preferred Reporting Items for Case reports in Endodontics (PRICE), an extension of the CARE guidelines, for the field of endodontics (10). In 2016, Agha et al. developed the Surgical CAse REport (SCARE) guidelines using a consensus-based approach (11). The SCARE statement tailored the CARE guidelines to surgical case reporting to help researchers recognize specific reporting requirements in surgery.

The CARE guidelines have been extended to many fields, but not yet to radiology. Thus, currently, case reports outline the imaging features and manifestations of a disease separately. Due to the rapidly growing number and inconsistent quality of case reports in radiology, and the inapplicability of some items (e.g., Item 9 on therapeutic interventions cannot be evaluated in radiology case reports), there is an urgent need to develop reporting guidelines that are tailored to radiology and meet the specific requirements of this field adequately.

Thus, our objective is to conduct a Delphi survey (The Delphi survey is a method of gathering the collective opinion of a group of experts on a particular topic) with experienced methodologists, content experts, journal editors, and possibly consumer representatives to identify reporting items and develop reporting guidelines for case reports in radiology—the CAse Report for Radiology (CARR) statement. We have registered the CARR on the Enhancing the QUAlity and Transparency Of health Research (EQUATOR) network. The EQUATOR network is an international initiative that seeks to improve the reliability and value of published health research literature by promoting transparent and accurate reporting and wider use of robust reporting guidelines (12). This article presents the protocol for developing these guidelines.

\section{Methods}

We plan to extend the existing CARE guidelines to radiological case reports. We will follow the steps recommended by the EQUATOR network to develop the CARR statement and checklist (12), and other extensions of CARE (9-11). The EQUATOR Network is an international initiative aimed at promoting the transparent and accurate reporting of health research studies to enhance the value and reliability of medical research literature, and more than 470 health research guidelines had been added to and displayed on it $(13,14)$. We will adapt and modify the methods as appropriate. Figure 1 illustrates the process of CARR statement development. Figure 2 details the timetable. The study started on August 1, 2021, and all 


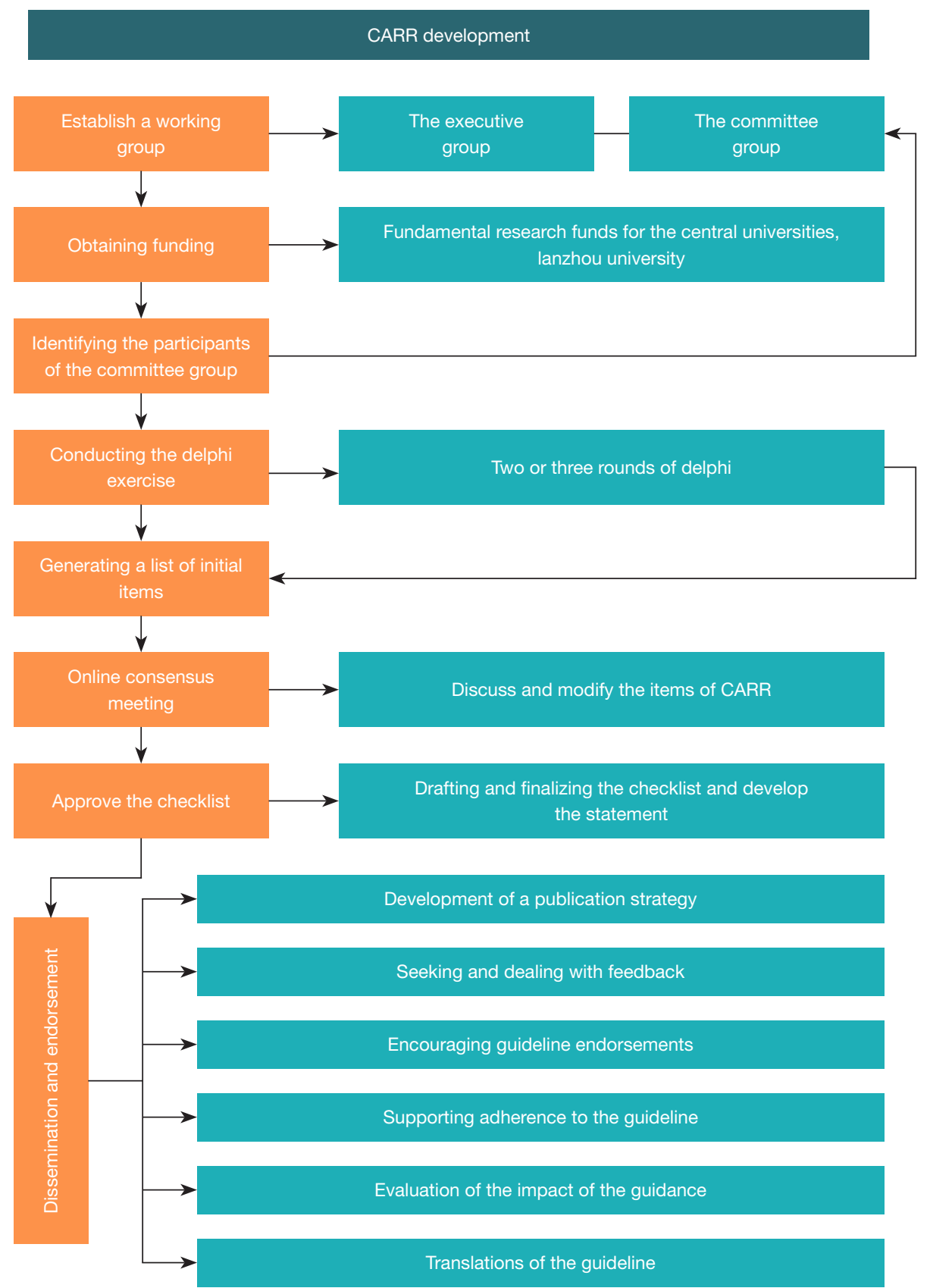

Figure 1 The process of CARR statement development. CARR, Case Report for Radiology.

work is scheduled to be completed by December 2022 .

\section{Establish a working group}

The CARR working group will consist of two subgroups: the executive group and the committee group. The executive group will be based at the Evidence-Based
Medicine Center of Lanzhou University and facilitate the development of the CARR statement in terms of the literature search, document preparation, organization of meetings, consensus process, and administration. The committee group will comprise experienced experts in relevant fields, including methodologists, content experts (radiologists and clinicians), journal editors, and possibly 


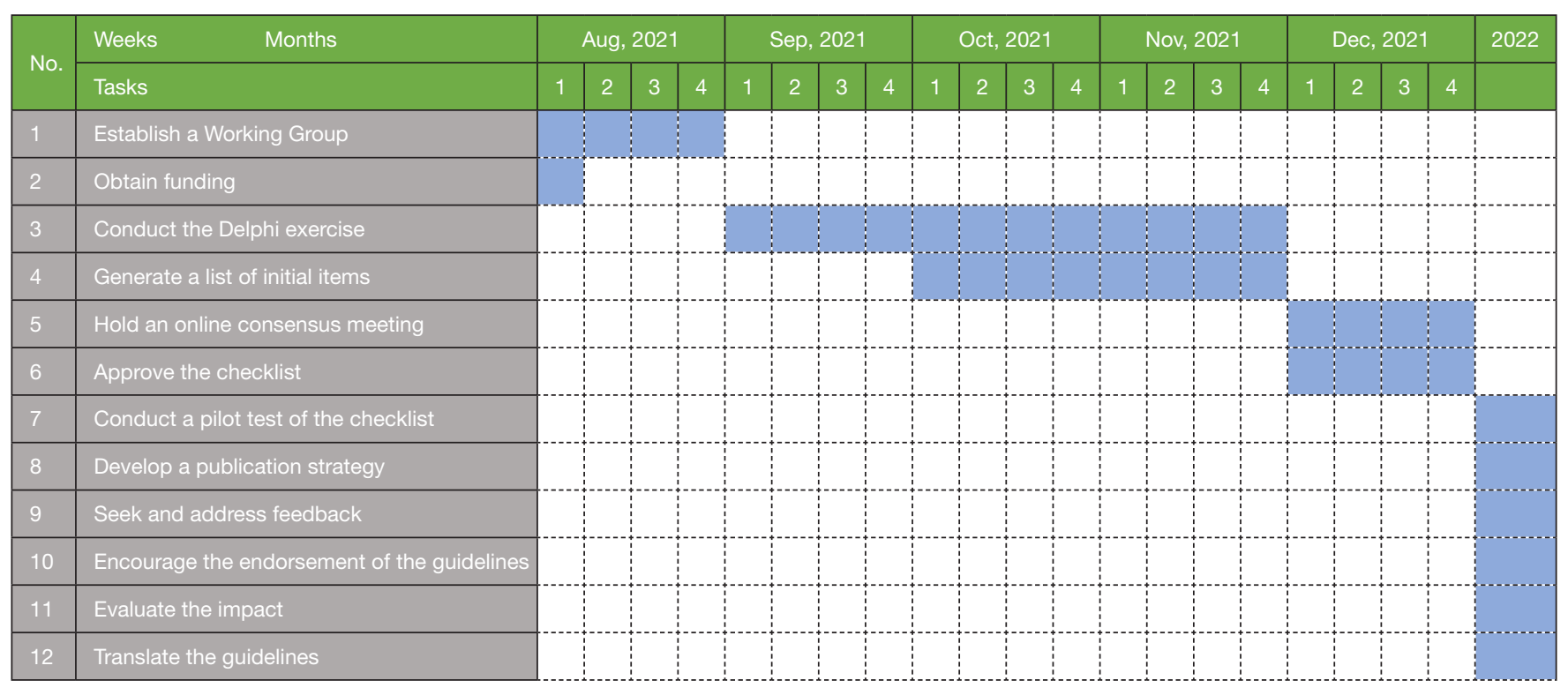

Figure 2 The timetable for the development of the CARR statement. CARR, Case Report for Radiology.

also consumer representatives. The committee group will participate in the identification of reporting items that are tailored to radiological case reports based on the CARE guidelines and reach a consensus on the final version of the CARR statement.

\section{Obtain funding for the initiative}

The funding will cover the expenses for pre-meeting activities, such as the literature search, meeting material preparation and the Delphi exercise, the working group meeting, and post-meeting activities, such as the drafting of the CARR guidelines, the writing of the CARR statement manuscript, and the development of the explanation and elaboration $(\mathrm{E} \& \mathrm{E})$ document. This work is funded by Fundamental Research Funds for the Central Universities, Lanzhou University (lzujbky-2021-ey13). The funding institution will play no role in the study design, data collection and analysis, writing of the article, or decisions to submit it for publication.

\section{Identify the members of the committee group}

The executive group will identify a list of potential participants for the committee group. The proposed committee group will be an international multidisciplinary team comprising 20-30 members, including clinical and pathological experts in the fields of radiology, guideline development, and case report publishing. We will also invite consumer or patient representatives to the committee group if possible. We will first send invitation emails to a small group of participants, who have participated in reporting guideline development groups at least once in the past year. This small group will include less than 10 people who have been responsible for the development of reporting guidelines, all of whom will be key members of the committee group. Next, we will ask each participant who accepted our invitation to suggest additional potential members (beyond those we identified in our list).

\section{Conducting the Delphi exercise}

We will administer the Delphi questionnaire through Survey-Monkey ${ }^{\circledR}$ (https://www.surveymonkey.com/) or LimeSurvey (https://www.limesurvey.org). We will start with a standard Delphi exercise and modify the methodology as appropriate. We plan to use the same questionnaire throughout the process, which means that the expert respondents will complete the questionnaire in all rounds of the Delphi exercise.

The executive group will design the questionnaire. The overall framework of the Delphi questionnaire will be based on the CARE statement and require the expert consensus of the committee group. In all rounds, the Delphi questionnaire will comprise two sections that cover the reporting items for radiological case reports, and sources of content for the 
adaptation and extension of CARE to radiology. In the first section, we will ask the respondents to rate the "importance" and "appropriateness" of each item in the CARE statement for radiology case reports. In the second section, we will ask the respondents to offer suggestions of new items or adaptations of the existing items in the CARE statement to fulfill the specific requirements of case reports in radiology.

\section{Statistical analysis}

The respondents will use a nine-point Likert scale to rate the importance and appropriateness of each reporting item on which a score of 1-3 indicates that the respondent disagrees that the item is of importance or appropriate for radiological case reports, 4-6 indicates that the respondent agrees that the item being rated is of importance or appropriate but not critical to radiological case reports, and 7-9 indicates that the respondent agrees that the item being rated is of importance or is appropriate and critical to radiological case reports. A consensus will be considered to have been reached if more than two thirds of the respondents give a score of 7 or above and less than one third give a score of 1 to 3 . We will revise items with scores of 4-6 based on the comments of the committee group members for the next round of the Delphi exercise. We will not restrict the number of rounds of the Delphi exercise. We anticipate that the participants will reach a consensus in two or three rounds. The use of online survey tools will guarantee the anonymity of other panel members' decisions.

\section{Generate a list of items for consideration at the virtual consensus meeting}

After each round of the Delphi process, the executive group will collect the completed questionnaires, compile the results, and summarize the respondents' comments and suggestions regarding any adaptations to the CARE guidelines and new items for the CARR statement. If no consensus is reached, we will continue the Delphi exercise for another round. Once a consensus is reached, the executive group will prepare a list of items for consideration by the committee group at the meeting.

\section{Prepare for the online consensus meeting}

\section{Venue and size}

Due to the Coronavirus Disease 2019 pandemic, travel restrictions have been imposed around the world, and it is not feasible to hold an in-person meeting. Thus, we will hold a virtual meeting via a teleconference platform (Zoom, Microsoft Teams, Skype, or Tencent Meeting).

\section{Duration}

We anticipate that the meeting participants will reach a consensus on the preliminary version of the CARR checklist in about one day. Thus, we intend to hold a one-day virtual meeting.

\section{Agenda}

The executive group will develop the agenda for the virtual meeting and circulate it in advance to all participants before the meeting. The overall framework of the agenda will include the CARR statement background material, a preliminary version of the CARR reporting checklist, the process of the virtual consensus meeting, and a discussion of the possibility of developing a flow diagram for the CARR statement.

\section{Present and discuss the results of pre-meeting activities and relevant evidence}

The executive group of the CARR working group will prepare and present the CARR statement background material, available evidence, and the rationale for the included items to the committee group before the beginning of the virtual meeting.

\section{Hold the virtual meeting}

The executive group will host the meeting. During the meeting, the executive group will facilitate the Delphi exercise, prepare the potential items, and provide background material as needed.

\section{Draft and finalize the checklist and develop the statement}

The CARR executive group will begin to draft the final checklist immediately after the virtual meeting. The executive group will also work in parallel on the CARR statement. In the CARR statement, we will explain which parts of the CARR checklist have been taken unchanged from the original CARE checklist and which items have been modified or added to respond to the specific requirements of radiology.

\section{Pilot test of the checklist}

We plan to conduct a pilot test of the CARR checklist among potential users for validation. The potential users 
include radiologists, clinicians and editors of journals publishing case reports in radiology, and we intend to collect their feedback on the checklist. The executive group will review the comments and incorporate them as appropriate into future revisions of the CARR checklist.

\section{Develop a publication strategy}

The executive group will facilitate the journal submissions. The executive group will work with the committee group to discuss the suitable journals, prepare a manuscript that all authors agree on, and submit the final manuscript to a set of journals for multiple simultaneous publications. The executive group will communicate with editors from suitable journals before submission to facilitate multiple publications. Additionally, the CARR checklist will be disseminated through multi-language channels.

\section{Seek and address feedback}

We will seek continuous feedback of any kind in relation to the methodology, reporting items or any other aspect of the CARR statement. We believe that constructive criticism will improve the quality of the CARR statement development process and enable us to identify items for future updates.

\section{Encourage the endorsement of the guidelines}

We will involve editors of radiological journals in the development of the CARR statement, which may facilitate the endorsement of the CARR statement by journals. We hope that the radiological journals that publish case reports will strongly recommend the use of the CARR statement in their guidance for authors and use clear language to describe their expectations regarding the requirements of radiological case reports.

\section{Support adberence to the guidelines}

We will continuously collect and review any feedback and criticism we received about the CARR checklist, and use this feedback and criticism to maximize adherence to the CARR statement by incorporating any critical feedback and criticism into revisions of the updated checklist.

\section{Evaluate the impact of the reporting guidelines}

We plan to conduct an impact evaluation of the guidelines.
The impact evaluation will focus on assessing the extent to which the CARR guidelines have improved the quality of reporting in radiological case reports.

\section{Translate the guidelines}

We plan to translate the CARR statement into Chinese and other languages if possible. The executive group will oversee the translations.

\section{Discussion}

Reporting guidelines for specific areas could enhance the quality of the corresponding research and promote its dissemination and transparency but will only do so if the methods used to develop them are scientific, disciplined and transparent. Our objective is to conduct a Delphi survey to identify reporting items and develop reporting guidelines for case reports in radiology. The CARR guidelines will be developed under the general requirements and methods of the international reporting guidelines (12). This article captures most of the methods and processes used to develop the CARR guidelines, but it still has some limitations. First, we did not conduct a systematic review to identify current reporting guidelines for radiology due to the paucity of study in this area, but we reviewed the methodologies, protocols, and content of the development of other extended versions of the CARE guidelines (9-11). Second, we did not hold a face-to-face meeting to consider the impact of the pandemic. Finally, we did not provide a detailed description of the expert group, as it has not yet been assembled. However, we do not believe that these limitations will affect the quality of the development of the CARR guidelines.

In line with other extensions of the CARE guidelines (9-11), we hope that the CARR guidelines will help imaging practitioners to improve the quality of case reports and promote greater transparency and clarity in their reporting.

\section{Acknowledgments}

We would like to thank Dr. Janne Estill for his comments on our article.

Funding: This work was funded by Fundamental Research Funds for the Central Universities, Lanzhou University (lzujbky-2021-ey13). The funding institution plays no role in the study design, data collection and analysis, writing of the article, or decisions to submit it for publication. 


\section{Footnote}

Conflicts of Interest: All authors have completed the ICMJE uniform disclosure form (available at https://atm. amegroups.com/article/view/10.21037/atm-21-7028/coif). The authors have no conflicts of interest to declare.

Ethical Statement: The authors are accountable for all aspects of the work in ensuring that questions related to the accuracy or integrity of any part of the work are appropriately investigated and resolved.

Open Access Statement: This is an Open Access article distributed in accordance with the Creative Commons Attribution-NonCommercial-NoDerivs 4.0 International License (CC BY-NC-ND 4.0), which permits the noncommercial replication and distribution of the article with the strict proviso that no changes or edits are made and the original work is properly cited (including links to both the formal publication through the relevant DOI and the license). See: https://creativecommons.org/licenses/by-nc-nd/4.0/.

\section{References}

1. Dragnev NC, Wong SL. Do we CARE about the quality of case reports? A systematic assessment. J Surg Res 2018;231:428-33.

2. Gagnier JJ, Kienle G, Altman DG, et al. The CARE guidelines: consensus-based clinical case report guideline development. J Clin Epidemiol 2014;67:46-51.

3. Lennon P, Fenton JE. The case for the case report: refine to save. Ir J Med Sci 2011;180:529-32.

4. Bannas P. Instructions for Radiological Case Reports. Rofo 2017;189:333-8.

Cite this article as: Wang M, Zhu Y, Luo X, Xiao X, Wang L, Lv M, Wang Q, Chen Y, Lei J, Tian J. Protocol for developing the reporting guidelines for radiological case reports: Case Report for Radiology statement. Ann Transl Med 2022;10(2):107. doi: $10.21037 / \mathrm{atm}-21-7028$
5. Chow DS, Ha R, Filippi CG. Increased rates of authorship in radiology publications: a bibliometric analysis of 142,576 articles published worldwide by radiologists between 1991 and 2012. AJR Am J Roentgenol 2015;204:W52-7.

6. Riley DS, Barber MS, Kienle GS, et al. CARE guidelines for case reports: explanation and elaboration document. J Clin Epidemiol 2017;89:218-35.

7. Elsevier. Radiology Case Report Author Information Pack. August 5, 2021. Accessed August 5, 2021. Available online: https://www.elsevier.com/wps/find/journaldescription. cws_home/736967?generatepdf=true

8. Hindawi. For authors. Hindawi. August 5, 2021. Accessed August 5, 2021. Available online: https://www.hindawi. com/journals/crira/guidelines/

9. Lavergne V, Ouellet G, Bouchard J, et al. Guidelines for reporting case studies on extracorporeal treatments in poisonings: methodology. Semin Dial 2014;27:407-14.

10. Nagendrababu V, Chong BS, McCabe P, et al. PRICE 2020 guidelines for reporting case reports in Endodontics: a consensus-based development. Int Endod J 2020;53:619-26.

11. Agha RA, Fowler AJ, Saeta A, et al. The SCARE Statement: Consensus-based surgical case report guidelines. Int J Surg 2016;34:180-6.

12. Moher D, Schulz KF, Simera I, et al. Guidance for developers of health research reporting guidelines. PLoS Med 2010;7:e1000217.

13. Simera I, Moher D, Hoey J, et al. A catalogue of reporting guidelines for health research. Eur J Clin Invest 2010;40:35-53.

14. Simera I, Moher D, Hirst A, et al. Transparent and accurate reporting increases reliability, utility, and impact of your research: reporting guidelines and the EQUATOR Network. BMC Med 2010;8:24. 